1895. Mille huit cent quatre-vingt-quinze

Revue de l'association française de recherche sur

I'histoire du cinéma

$61 \mid 2010$

Aux sources du burlesque cinématographique : les comiques français des premiers temps

\title{
Une « atmosphère de nursery du diable ». Pantomime de cirque et premier cinéma comique
}

\section{Patrick Désile}

\section{(2) OpenEdition}

1 Journals

Édition électronique

URL : https://journals.openedition.org/1895/3832

DOI : 10.4000/1895.3832

ISBN : 978-2-8218-0980-2

ISSN : 1960-6176

Éditeur

Association française de recherche sur l'histoire du cinéma (AFRHC)

Édition imprimée

Date de publication : 1 septembre 2010

Pagination : 114-127

ISBN : 978-2-913758-62-9

ISSN : 0769-0959

\section{Référence électronique}

Patrick Désile, «Une « atmosphère de nursery du diable ». Pantomime de cirque et premier cinéma comique », 1895. Mille huit cent quatre-vingt-quinze [En ligne], 61 | 2010, mis en ligne le 01 septembre 2013, consulté le 17 avril 2022. URL : http://journals.openedition.org/1895/3832 ; DOI : https:// doi.org/10.4000/1895.3832 
Nouveau Irque

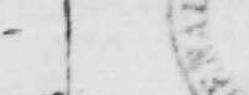

Tous les Sorre

a $8 \mathrm{H} / 2$

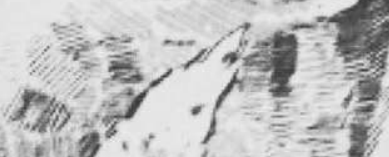

5. $100^{2}$.

y-
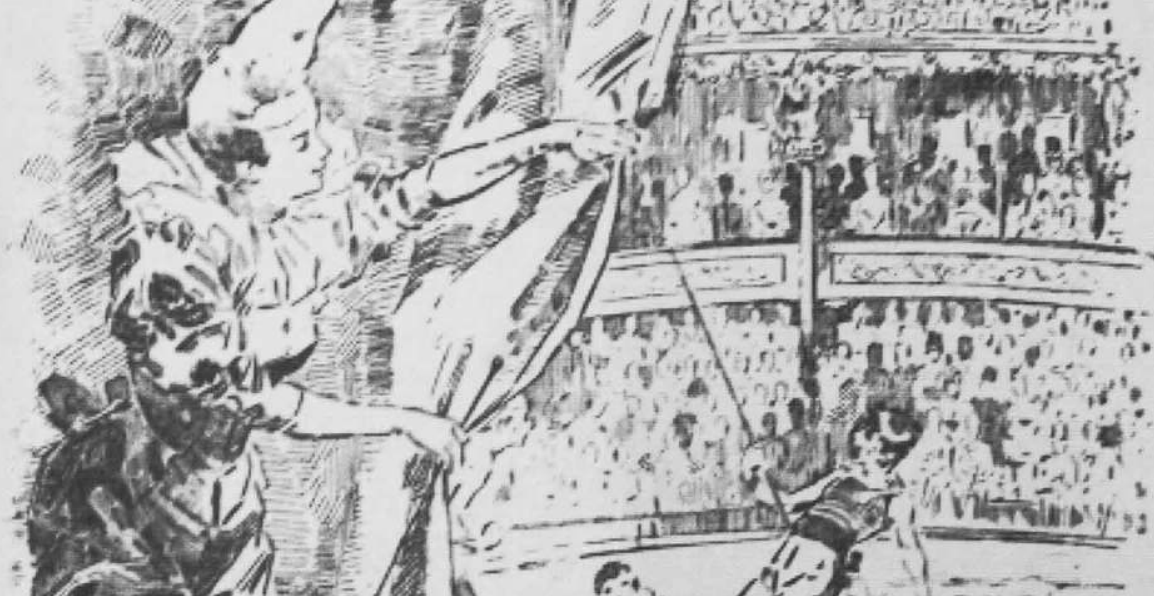

1)

R̂
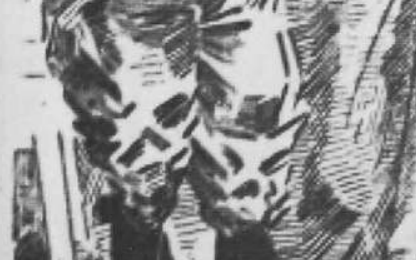

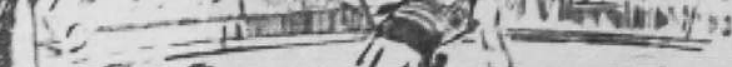
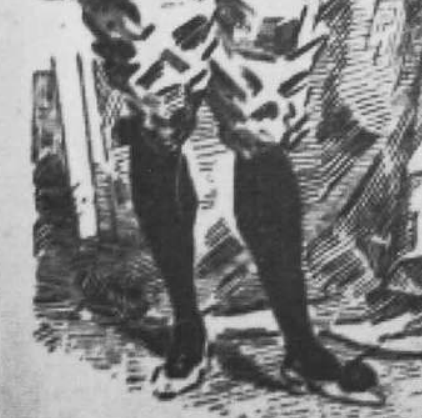

3
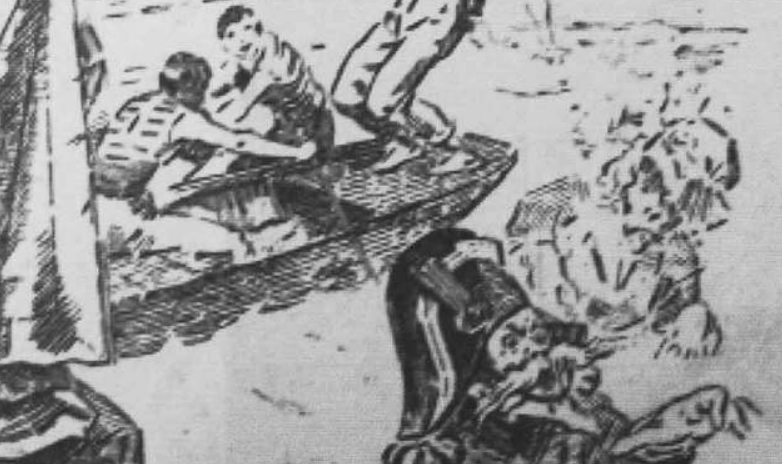

Prix : 20 Centimes

MERCREDIS, JEUDIS, OIMANCHES \& FËTES. MATINÉES a 2 ":\%

Programme du Nouveau Cirque, 27 février 1898. 


\section{Une " atmosphère de nursery du diable ". Pantomime de cirque et premier cinéma comique}

Par Patrick Désile

... Foottit apportait sur la piste une atmosphère de nursery du diable, où les enfants retrouvaient leurs malices sournoises et dont les grandes personnes subissaient la grandeur. Jean Cocteau, Portraits-souvenir, 1935

La généalogie du premier cinéma comique français se résumerait ainsi : les toutes premières bandes comiques, ou du moins beaucoup d'entre elles, seraient inspirées de dessins humoristiques ou de numéros de cirque ou de music-hall, en seraient même souvent la reprise. Plus tard, les films de poursuites seraient imités du cinéma anglo-saxon, lui-même empruntant sans doute certains éléments à la pantomime anglaise ou au vaudeville américain, mais fondant, cependant, un genre de spectacle nouveau.

De manière plus générale, le premier cinéma comique français serait aussi redevable aux domaines du cirque, du music-hall, de la fête foraine : ses acteurs, d'abord, en seraient souvent issus, clowns ou acrobates multipliant les cabrioles et important une gestuelle. Surtout, populaire comme eux, le spectacle cinématographique en adopterait le caractère segmenté, hétéroclite, heurté.

Le reste serait à mettre au compte de l'inventivité des premiers cinéastes.

Sans nier toute validité à ce schéma, je voudrais I'amender en montrant ce que le premier cinéma comique français, particulièrement sous sa forme «burlesque ", doit, pour la forme et pour les contenus, à un genre de cirque assez généralement ignoré, la pantomime française de cirque.

J'entends seulement ici établir l'existence de relations entre ces deux formes de spectacle et 
$1895 /$

commencer à les spécifier, me réservant de revenir plus tard, d'une part sur ce qu'elles impliquent pour l'élaboration des modes de représentation cinématographique, d'autre part sur l'interprétation d'un certain nombre de figures communes aux deux univers, que je me bornerai ici à décrire sommairement.

Mais il importe de procéder d'abord à quelques mises au point. Les discours ordinaires sur le cirque, quelquefois même les discours savants, sont assez généralement mal informés et souvent nourris de fantasmes (il en va de même pour le café-concert, le music-hall et les spectacles forains). Si certaines études sur le premier cinéma, notamment, ne manquent pas d'indiquer sa proximité avec "le cirque, le music-hall, la fête foraine ", l'emploi de ce syntagme trinitaire figé, incantatoire et comme automatique (où " music-hall » est parfois remplacé par "café-concert ", il est vrai) paraît souvent tenir lieu de savoir. On semble faire comme si ces spectacles avaient fait l'objet de travaux abondants, précis, fiables, comme si ces travaux étaient connus de tous, comme s'il était par conséquent inutile d'en dire davantage. Mais de tels travaux sont fort peu nombreux et peu diffusés (je parle ici du domaine français), et ces spectacles ne sont donc ni bien connus ni pensés. Peut-être, obscurément, ne les juget-on d'ailleurs pas tout à fait dignes de l'être ? En tout état de cause, si l'on bâtit des hypothèses à leur sujet, c'est souvent sur du sable.

Que veut-on dire, par exemple, en subsumant ces spectacles (et quelques autres) sous la catégorie des "spectacles populaires " ? Veut-on signifier qu'ils sont fréquentés seulement ou principalement par des catégories sociales que l'on peut dire "populaires » ? Admettons résolue la question de leur définition, il reste qu'on ne possède à peu près aucune donnée statistique sur les publics. Se fondera-t-on, alors, sur des témoignages ? Mais il faudrait les analyser comme tels, et il se trouve de toute façon que, s'agissant par exemple du cirque à la fin du XIXe siècle, ils attesteraient plutôt une fréquentation s'étendant à l'ensemble des catégories sociales (cela devrait être nuancé, autant que faire se pourrait, en fonction des lieux et des types de cirques, bien entendu). Pour certains cirques parisiens, ils montrent même une fréquentation habituelle par les catégories sociales supérieures (et pas seulement par une élite intellectuelle ou artistique). Peut-on qualifier tout uniment de « populaire » un spectacle dont les représentations sont régulièrement évoquées dans la Revue mondaine illustrée, entre la "Chronique mondaine » de la comtesse Paule et la rubrique "Finances » de Pierre Largentier 1 , ou dont il est longuement rendu compte dans la Revue blanche sous la plume d'Alfred Jarry² ? Mais peut-être veut-on suggérer que ces spectacles doivent être

1 Voir par exemple la Revue mondaine illustrée, 15 avril 1893.

2 Alfred Jarry, "Gestes. Juno Salmo au Nouveau Cirque », la Revue blanche, t. XXVII, janvier-avril 1892, pp. 146-149. 
considérés comme " populaires ", parce qu'ils seraient, par exemple, simples, visuels, physiques... Mais on voit bien qu'on est là dans l'ordre des représentations, sinon dans celui des fantasmes, et que tout cela demanderait à être déplié, explicité, analysé, ce qui, pour autant qu'on le sache, ne se fait guère. Mieux vaut donc se garder d'user de l'encombrante catégorie de "spectacle populaire ", qui, loin de favoriser l'intelligibilité, y fait obstacle.

Si maintenant nous en venons à la pantomime de cirque, plusieurs équivoques doivent encore être dissipées. Un spectacle de cirque, au XIXe siècle, comporte très généralement deux parties. Dans la première sont présentés, en une suite en effet hétérogène, des numéros équestres, ou d'acrobatie, de dressage, etc. La seconde est la pantomime, qu'on peut parfaitement assimiler à une pièce de théâtre. À Paris, le Cirque olympique pendant les premières décennies du siècle, le cirque Fernando ou le Nouveau Cirque au cours des dernières, donnent ordinairement des pantomimes. Les hippodromes, qui sont de vastes cirques, en présentent aussi, de même que le Cirque d'hiver et le Cirque d'été, ceux-là plus rarement, il est vrai. Les pantomimes ne sont d'ailleurs pas réservées aux cirques stables, les cirques itinérants les inscrivent aussi à leur programme, du moins dans les dernières décennies du siècle ${ }^{3}$. La pantomime n'est donc nullement un épiphénomène, c'est une des composantes majeures du spectacle de cirque au XIXe siècle, au début du XXe siècle encore.

En quoi consiste-t-elle ? On fait parfois mine de croire, pour autant, du moins, qu'on l'évoque, qu'elle serait une sorte de spectacle de mime à la Deburau, en plus clownesque. Ce n'est nullement le cas. Le mot " pantomime ", au XIXe siècle, a des emplois divers, complexes, et il peut recouvrir des genres théâtraux notablement différents. La pantomime n'est pas nécessairement le mime, et elle est loin d'être toujours muette. À côté de la pantomime proprement dite, la pantomime dialoguée est d'ailleurs un genre établi, que l'auteur et critique dramatique Charles-Maurice Descombes évoque en ces termes, en 1860 :

\author{
En offrant à la fois \\ Le silence prodigue et l'avare langage, \\ II [ce spectacle] versait l'ambroisie au Public de son choix \\ Qui n'en voulait pas davantage ; \\ C'eut été de l'abus. \\ [...] \\ La source était féconde et chacun y puisait,
}

3 Les dispositions juridiques se rapportant aux cirques et au genre de spectacles qu'ils étaient autorisés à présenter sont complexes, et elles ont varié dans le temps et dans l'espace (longtemps, le Cirque d'été n'a pas été autorisé à présenter de pantomimes, par exemple, et il en allait très souvent de même pour les cirques itinérants). Les exposer imposerait des développements fastidieux et sans véritable utilité ici. 
La Gaîté, la Cité, l'Ambigu, jusqu'au Cirque,

En ont usé $[\ldots]^{4}$

Les pantomimes représentées dans les cirques peuvent être entièrement mimées, elles peuvent faire alterner des passages mimés et des passages dialogués, elles peuvent enfin, et c'est très souvent le cas, être entièrement dialoguées, comme l'est une pièce de théâtre ordinaire. Seulement ce sont presque toujours des pièces « à grand spectacle ", qui usent de nombreux effets visuels : acrobaties, prouesses équestres, intervention d'animaux sauvages, effets pyrotechniques, trucs, etc.

Pour lever toute ambiguïté, il faut encore préciser deux choses. Les pantomimes sont de véribien écrites. Elles auraient pu être représentées sur une scène de théâtre, et l'ont parfois été, en effet. Le Cirque olympique combinait, d'ailleurs, une piste et une scène et il était considéré comme un théâtre. II ne s'agit donc nullement de vagues canevas qui serviraient à faire le lien entre des numéros de cirque. Mais si ces pantomimes sont pleinement du théâtre, elles sont aussi pleinement du cirque. Les prouesses sont intimement intégrées à la trame narrative, mais elles sont réalisées par de véritables artistes de cirque, généralement par ceux-là même qui ont exécuté les numéros de cirque pur de la première partie. Il y a ainsi, dans la pantomime de cirque, deux pôles, celui du théâtre et celui du cirque, ou, si l'on veut, celui de la narration et celui de l'attraction, entre lesquels les auteurs réussissent généralement à maintenir un équilibre. Et s'il est vrai que les pièces des dernières décennies du siècle, dont nous allons surtout parler, sont souvent des farces, en partie improvisées, dans lesquelles le scénario importe moins que les effets visuels, il n'est pourtant jamais un simple prétexte. On aperçoit donc déjà, d'après cet exposé liminaire, que la pantomime de cirque, riche d'une expérience séculaire, peut avoir constitué un modèle, parmi d'autres, conscient ou non, pour le cinéma en train de se construire, modèle qu'on pourrait ainsi décrire : une trame narrative qui intègre des moments d'attraction, une continuité qui maintient l'attention, et des sortes de coups sensoriels qui suscitent et relancent l'intérêt. Si ce modèle n'appartient pas à la seule pantomime de cirque, c'est sans doute là que l'équilibre entre les deux pôles est le mieux assuré.

Cela posé, il convient d'essayer de repérer des relations entre ces pantomimes et le premier cinéma comique et de les décrire. Je mentionnerai quelques pantomimes du Cirque olym-

4 Charles-Maurice Descombes, Tous les genres de théâtre et sujets correspondants : épîtres en vers libres, Paris, impr. De Serrière, 1860, pp. 64-65. 
pique $^{5}$ et du cirque Fernando 6 , mais j'évoquerai surtout celles du Nouveau Cirque7, ainsi que quelques revues (pantomimes qui prennent pour sujet l'actualité de l'année écoulée et dont le texte est généralement plus élaboré). Pour ce qui est des films, j'envisage ici l'ensemble de la production comique de 1895 à 1914, l'espace d'un article ne me permettant pas de distinguer des catégories qu'un exposé plus ample devrait prendre en compte, bien entendu. Pour ce qui est des relations entre les deux domaines, enfin, je laisserai de côté les relations externes, en particulier les relations d'ordre social, disons même biographique (je pense à des transfuges qui seraient passés d'un univers à l'autre) pour m'attacher aux contenus et à la forme des spectacles.

La première figure que j'évoquerai est très présente dans le premier cinéma. On la rencontre souvent dans les films de Georges Méliès (Un homme de tête, 1898, I'Homme à la tête de caoutchouc, 1901, le Mélomane, 1903...), mais on la trouve aussi dans d'autres films, comme le Barbier fin-de-siècle (Pathé, 1896) ou Chez le dentiste (Pathé, 1901) : cette figure, c'est la décapitation comique, qui s'accompagne, généralement, du retour de la tête à sa place. Contrairement à ce que l'on pourrait peut-être penser, elle n'est pas particulièrement fin-desiècle, et elle n'est pas proprement cinématographique : parmi les premiers trucs cinématographiques, il en est peu qui ne soient la transposition de trucs de théâtre. La décapitation comique traverse à vrai dire le siècle. Elle est déjà présente dans des féeries du Cirque olympique : Zazezizozu8 en 1835 (après que le prince Zazezizozu a fait couper la tête de son astrologue, elle demande à être entendue, puis elle intervient à différentes reprises avant de retrouver sa position initiale) ou les Pilules du diable9 en 1838 (le grand rasoir qui sert d'enseigne à un barbier se détache et tranche la tête du valet Magloire ; il la retrouve plus tard, mais elle est mal fixée sur ses épaules).

La décapitation est aussi la figure centrale du Frater de village ${ }^{10}$, qui fut la première et longtemps la seule pantomime des frères Hanlon Lees. Bien que les pantomimes des Hanlon Lees ne soient pas à proprement parler des pantomimes de cirque, puisqu'ils les ont plutôt représentées sur scène (notamment aux Folies Bergère et au théâtre des Variétés), il est impossible de ne pas les évoquer, parce que leur comique acrobatique, délirant et cruel, qui obtint

5 Le Cirque olympique, créé en 1807, seul établissement parisien jusqu'en 1835, a donné de nombreuses pantomimes militaires, mais aussi des pantomimes comiques, comme certaines féeries.

6 Le cirque Fernando, d'abord simple chapiteau installé par Ferdinand Beert en 1873 à l'angle de la rue des Martyrs et du boulevard Rochechouart, est devenu cirque stable en 1875. Il fut repris par le clown Medrano en 1897.

7 Le Nouveau Cirque a été ouvert en 1886 par l'entrepreneur de spectacles Joseph Oller, rue Saint-Honoré. C'est un cirque particulier dans la mesure où la piste pouvait se transformer en piscine.

8 Zazezizozu, féerie vaudeville, Cirque olympique, 1835, Archives nationales, F 181324.

9 Les Pilules du diable, féerie, Cirque olympique, 1838, Archives nationales, F 181325.

10 Mémoires et pantomimes des frères Hanlon Lees, 1879, p. 109. 
tant de succès à Paris dans les années 1870, a considérablement influencé celui des pantomimes du cirque Fernando ou du Nouveau Cirque (dont le régisseur général, Agoust, avait été le partenaire des Hanlon Lees). Un frater, c'est-à-dire l'aide d'un barbier-chirurgien, entreprend de raser les membres d'une famille avec un gigantesque rasoir ; les têtes sont coupées, puis recollées.

On trouve aussi la décapitation dans la pantomime du Nouveau Cirque Boule de Siam, en 1894. Un serpent arrache la tête d'un anglais : "Ce n'est rien, ça m'est arrivé trois fois ! "11 dit un autre personnage. On recolle la tête, en effet, aussitôt. Quant au Barbier12, pantomime « du vieux cirque » recueillie par Raoul Donval (lui-même auteur de pantomimes et, un temps, directeur du Nouveau Cirque), elle met en scène un barbier qui, bousculé, tranche involontairement la tête d'un " bourgeois ", puis celle d'un autre client, pour qu'il ne s'aperçoive de rien. Plus tard, les têtes sont pourtant replacées sur les corps, mais, interverties, elles ne les animent pas. Enfin, tout rentre dans l'ordre. Cette pantomime comporte bien d'autres péripéties, et tout se passe comme si le Barbier fin-de-siècle, dans lequel le barbier ôte la tête d'un client pour la raser, y avait seulement prélevé un instant décisif.

La figure de la décapitation comique, dont le contenu symbolique est évidemment très riche, est relativement pérenne. D'autres figures, dont la complexité n'est peut-être pas moindre, ont des rapports plus étroits avec les circonstances. Parmi elles, je citerai l'ensemble de celles qui, liées aux représentations, fantasmatiques et ambivalentes, de l'Amérique, laissent paraître une fascination, où se mêlent angoisse et jubilation, pour la modernité brutale et pour la sauvagerie supposée du Nouveau Monde. L'une de ces figures se rencontre, au cinéma, dès 1895, dans le film Lumière Charcuterie mécanique (titré aussi Charcuterie américaine) : un porc est introduit vivant dans la machine de la charcuterie Craque, dont ne tardent pas à sortir des saucisses. Alice Guy donne, en 1900, une version plus insolite et plus caustique de ce film avec Chapellerie et charcuterie automatiques, dans laquelle ce sont des chats qui constituent la matière première des saucisses, mais aussi des chapeaux que produit la machine. Le film de 1895 était sans nul doute inspiré par les descriptions des abattoirs de Chicago, comme celle, élégante et terrifiante, que publia Paul Bourget après la visite qu'il en fit en 189313. Elles donnèrent lieu à diverses extrapolations humoristiques, dans lesquelles, comme dans le film Lumière, un cochon vivant introduit dans la machine ressortait aussitôt

11 Boule de Siam, exhibition d'éléphants, Nouveau Cirque, 1893, Archives nationales, F 181327. 12 "Le Barbier ", dans Pantomimes du Vieux Cirque, recueillies et publiées par R. Donval, Directeur du Nouveau Cirque, Saint-Valéry en Caux, Imp. Eugène Dangu, 1896, pp. 117-129.

13 Publiée d'abord dans la presse américaine, sa relation parut en France en 1895 sous le titre Outre-mer. Notes sur l'Amérique, en deux volumes, chez Alphonse Lemerre. La description des abattoirs de Chicago occupe les pages 164 à 177 du volume 1. 
sous forme de charcuterie. En septembre 1896, la Revue encyclopédique pouvait indiquer : « Tout le monde a entendu parler de cette merveilleuse machine qui fonctionne, dit-on, à Chicago " ${ }^{14}$. Mais l'article, pourtant bref, qui commençait ainsi montrait que le thème était plus riche et plus troublant que la seule vignette cinématographique, facétieuse, ne le laissait peutêtre supposer. II mentionnait d'abord la possibilité d'inverser le processus en faisant machine arrière, et donc de ressusciter le cochon - mais cette possibilité était aussi, après tout, offerte par le cinématographe, frère de lait de la charcuterie Craque, et, par ce biais, apparenté fantasmatiquement aux abattoirs industriels. L'article était illustré par la reproduction d'un dessin humoristique allemand ${ }^{15}$ dans lequel, au cours d'une démonstration de la machine, un des spectateurs y tombait et était transformé en charcuterie ; une automatisation aveugle et meurtrière ravalait ainsi l'homme au rang de viande hachée, de matière première. Enfin le texte de la Revue encyclopédique affirmait que la réalité n'était " guère inférieure " à la légende en décrivant deux machines authentiques, européennes, l'une de fabrication de chaussures, l'autre de papier : I'imaginaire ne faisait donc peut-être qu'anticiper le réel.

Si j'ai voulu montrer un peu la complexité de cette inquiétante figure, c'est que, si elle n'apparaissait guère dans le film Lumière, elle se manifestait bien davantage, et dès janvier 1895, dans une pantomime du Nouveau Cirque, América ${ }^{16}$. Au Texas, un inventeur faisait la démonstration d'une machine qui, ayant produit de la charcuterie à partir d'un cochon vivant, recréait le cochon vivant à partir de la charcuterie. Mais cette machine était aussi capable, après avoir déchiqueté la belle-mère de l'inventeur, de la reconstituer à partir des morceaux épars. Elle sortait de là « évanouie, mais raccommodée «17.

Les représentations, directes ou indirectes, de l'Amérique par le premier cinéma comique ne se bornent évidemment pas aux deux bandes que j'ai mentionnées. Les incursions de Calino ou d'Onésime au Far-West (Calino veut être cow-boy, 1911, Onésime sur le sentier de la guerre, 1913, Onésime dresseur d'hommes et de chevaux, 1913, tous films de Jean Durand pour Gaumont) y renvoient explicitement mais ne sont peut-être pas les plus révélatrices. Les thèmes du rythme syncopé, de la danse trépidante, qu'ils soient contagieux (la Bous bous mie, Louis Feuillade, Gaumont, 1909) ou imposés (le Cake-walk forcé, Pathé, 1907), sont, en partie au moins, liés à l'introduction en France de musiques et de danses exotiques, dont le cake-walk, dansé au Nouveau Cirque en 1903, est la plus notoire18. Mais la contagion du

14 La Revue encyclopédique, Paris, Larousse, 19 septembre 1896, n¹59, p. 151.

15 "Expérimentation d'une machine à saucisses », Almanach des Fliegende Blaetter, 1894, Munich, reproduit in ibid.

16 América, fantaisie comique, Nouveau Cirque, 1895, Archives nationales F 181327.

17 lbid.

18 Sur tout cela, voir Rae Beth Gordon, Why the French Love Jerry Lewis : From Cabaret to Early Cinema, Stanford University Press, Stanford, 2001.

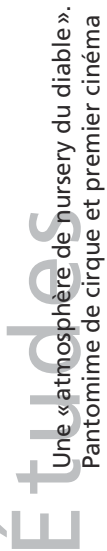


rythme, liée à l'image d'une Amérique vibrante, à la fois mécanique et instinctive, est présente au Nouveau Cirque dès 1895 dans América ${ }^{19}$, quand deux "policemen ", observant, dans un bar, deux « nègres ${ }^{20}$ qui dansent « finissent par s'en aller en dansant aussi », ou quand, un peu plus tard, les dames, qui se sont lancées dans une "Skirt-Dance ", sont bientôt suivies par toute l'assistance. Elle l'est aussi dans la Petite Revue ${ }^{21}$ du Nouveau Cirque en 1905, quand les airs de Faust qu'entonne la Garde républicaine de retour de l'exposition de Saint-Louis se muent en cake-walk et que les musiciens, puis l'Américain Crockett et tous les protagonistes se mettent à le danser. « C'est épouvantable ! lance le chef d'orchestre. Depuis qu'ils ont été en Amérique, voilà comment ils jouent Gounod et Wagner ! Cannibales !... Peaux Rouges !... »22 Une autre figure, partiellement liée, d'ailleurs, aux représentations de l'Amérique, figure centrale au Nouveau Cirque dans les années 1890 à 1910 et récurrente dans le premier cinéma comique, c'est celle de l'opposition du blanc et du noir, particulièrement de l'homme blanc et de I'homme noir. Cette figure est essentielle au Nouveau Cirque, puisque Foottit et Chocolat, qui y triomphèrent alors, en particulier dans les pantomimes, étaient l'un, blanc souvent vêtu de clair, l'autre, noir vêtu d'un frac, et que tout opposait leurs personnages, le premier cynique et manipulateur, le second naïf et résigné. Chocolat, insulté, battu, berné est perpétuellement infériorisé. Dans Coco, il interprète le prince Coco, qu'un gardien du jardin d'acclimatation prend d'abord pour un singe. On doit donc, à l'évidence, associer cette figure à l'émergence d'un racisme explicité (les mots raciste et racisme apparaissent précisément dans ces années-là), mais c'est plus largement la question de l'Autre qui paraît être ici anxieusement posée ${ }^{23}$. Cette opposition recèle en effet des ambiguïtés qui sont souvent des ressorts du comique : Foottit est odieux et vêtu comme un enfant, Chocolat avenant et vêtu comme un homme du monde. D'autre part, une inversion du noir et du blanc (qui s'accompagne parfois d'une inversion sexuelle) est souvent envisagée, parfois réalisée. On se souvient de la question, à nos yeux stupéfiante, que pose Bergson en 1899 dans la série d'articles de la Revue de Paris qui deviendront le Rire : " ... pourquoi rit-on d'un nègre ? "24. Question " embarrassante », mais peut-être résolue, dit Bergson, " par un simple cocher, qui traitait de " mal lavé " le client nègre assis dans sa voiture ${ }^{25}$. C'est ce ressort de l'inversion qui est uti-

19 América, op. cit.

20 « Le nègre et le policeman sont [...] deux des personnages favoris " des " farces à gros succès populaires " du théâtre américain (Paul Bourget, Outre-Mer, op. cit., vol. 2, p. 162).

21 Petite Revue, Nouveau Cirque, 1905, Archives nationales F 181327.

22 lbid.

23 Sur Chocolat, voir aussi Rae Beth Gordon, Dances with Darwin, Farnham, Ashgate, 2009, pp. $159-163$.

24 Henri Bergson, le Rire. Essai sur la signification du comique, Paris, P.U.F., 1940 [1899], p. 31.

25 lbid. 
lisé dans Boule de Siam²6: Chocolat ne peut épouser la jeune fille qu'il aime à cause de la couleur de sa peau, deux Européens réussissent à le « blanchir par un procédé américain ». Dans Paris qui trotte27 (1898), Chocolat surgit d'une caisse "vêtu en dame blanche ". Dans le Feu au moulin ${ }^{28}$ (1896), le baron de Saint-Gaga croit rencontrer la meunière qu'il courtise, mais il s'agit de Chocolat et de ce quiproquo résulte une "scène comique ".

Le premier cinéma comique use aussi volontiers des ces figures d'opposition et d'inversion. Les six films Lumière où apparaissent Foottit et Chocolat, captations de moments empruntés à leurs numéros, ne montrent guère que leur antagonisme. Mais dans le Rémouleur et l'assiette au noir (Lumière, 1897), un homme, à son insu, se noircit le visage avec du noir de fumée. Dans le Nègre gourmand (Pathé, 1905), un homme noir mange gaiement la crème Chantilly d'un gâteau en s'en barbouillant un peu, jusqu'à ce que surgisse un homme blanc qui, en colère, saisit le gâteau et le lance au visage de I'homme noir, le rendant ainsi blanc. Dans Un rêve de Dranem (Pathé, 1906), la femme blanche que Dranem croit étreindre devient tout à coup noire. Dans Zigoto et la blanchisseuse (Gaumont, 1912), Zigoto, poursuivi, plonge avec son chien dans le bac d'amidon de la blanchisserie, qui les rend blancs, puis dans le bleu de lessive qui les rend noirs aux yeux du spectateur.

Bien d'autres figures communes à la pantomime de cirque et au premier cinéma comique pourraient être repérées. J'en donnerai encore, plus rapidement, quelques exemples, pour montrer leur profusion.

La figure du déshabillage impossible est récurrente au cinéma (Lahury réserviste, Pathé, 1901 ; Comment Monsieur prend son bain, Gaumont, 1903...) ; mais dans la Noce à Chocolat, en 1888 , déjà, un homme qui " se met à se déshabiller précipitamment " 29 " enlève force vêtements sans en finir jamais "30. Arlequin, jaloux, joue des tours à Pierrot grâce à une flûte enchantée (Pierrot et la flûte enchantée, Lumière, 1898) qui n'est pas très différente de celle qu'utilisait l'amoureux éconduit de la pantomime pour faire danser les importuns (la Flûte enchantée $\left.{ }^{31}\right)$. La statue animée de la Statue (Gaumont, 1905), d'Hallucination de Pierrot (Pathé, 1906), ou de l'Homme de marbre (Gaumont, 1907) figure déjà dans les pantomimes recueillies par Raoul Donval, le Songe d'or32 ou Arlequin statue ${ }^{33}$. Onésime passe d'un esta-

26 Boule de Siam, op. cit.

27 Paris qui trotte, revue à grand spectacle, Nouveau Cirque, 1898, Archives nationales F 181327.

28 Le Feu au moulin, paysannerie nautique, Nouveau Cirque, 1896, Archives nationales F 181327.

29 Raoul Donval, la Noce à Chocolat, 1888, dans la Grenouillère, Saint-Valéry-en-Caux, E. Dangu, 18861893, p. 17.

30 lbid., p. 18.

31 « La Flûte enchantée », dans Pantomimes du Vieux Cirque, op. cit., p. 197.

32 "Le Songe d'or ", dans ibid., p. 9.

33 « Arlequin statue », dans ibid., p. 47. 
$1895 /$ figures essentielles des pantomimes des Hanlon Lees. Vers la fin de Pierrot terrible, par exemple, "c'est un tumulte indescriptible et une suite de poursuites à troubler l'imagination »38. Dans les Cascades du diable, « des gens [...] jouent à se casser des planches sur la tête. Pierrot veut en être ; il tape si fort que la maison d'en face est prise de delirium tremens et tombe en poussière " ${ }^{39}$. Dans Une soirée en habit noir, "glaces, rideaux, lustres, chaises, fauteuils, tout cela est broyé par la joie folle des invités ; la maison en tremble jusque dans ses fondements "40. Dans le Voyage en Suisse, enfin, le plafond s'écroule et les pierrots tombent sur une table où sont attablés des convives ${ }^{41}$. On croit entrevoir déjà le comique destructeur de certains films de Jean Durand.

Poursuites et cascades sont aussi récurrentes dans les pantomimes de cirque. Dès 1879, Une noce à roulettes ${ }^{42}$, au cirque Fernando, se présente comme une série de poursuites ; I'amou-

34 Pierrot aux Enfers, pantomime, Nouveau Cirque, 1897, Archives nationales F 181327.

35 Les Indiens Sioux, grande pantomime équestre et nautique, Nouveau Cirque, programme du 18 février 1900.

36 La Noce de Chocolat, Nouveau Cirque, programme du 17 juin 1894 (la Noce de Chocolat est la reprise de la Noce à Chocolat de 1888).

37 Une cascade, au théâtre, est aussi une plaisanterie (qui peut rester ignorée du spectateur) qu'un acteur ajoute à son rôle.

38 Mémoires et pantomimes des frères Hanlon Lees, op. cit., p. 123.

39 Ibid., p. 141.

40 Ibid., p. 161.

41 Ibid., p. 177.

42 Une noce à roulettes, pantomime, cirque Fernando, 1879, Archives nationales F 181327. 
reux éconduit par la famille est d'abord poursuivi par toute la noce, puis par les gendarmes ; le futur marié poursuit des enfants, il est lui-même poursuivi, ainsi que sa future bellemère, par les pompiers, puis par un ours ; I'amoureux est de nouveau poursuivi par les gendarmes, par les pompiers et par tous les protagonistes... « Poursuites et cascades pour tout le monde ${ }^{43}$ précise le manuscrit à tel moment de l'action.

Poursuites ou cascades, et souvent les deux, sont aussi indiquées dans les scénarios de nombreuses pantomimes du Nouveau Cirque, par exemple dans Gribouille44 (1891), dans le Roi Dagobert45 (1891), dans América46 (1895), dans Pierrot soldat47 (1895), dans la Reine de Bercy 48 (1895), dans la Petite Plage 49 (1895), dans le Feu au moulin ${ }^{50}$ (1896), dans Paris-Pékin ${ }^{51}$ (1896), dans Pierrot aux Enfers 52 (1897), dans les Cent kilos 53 (1897), dans Paris-Poursuites ${ }^{54}$ (1903), ou dans ceux de revues comme Paris qui trotte55 (1898)...

Il est évidemment difficile d'évaluer l'ampleur de ces poursuites et de déterminer précisément la nature de ces cascades : les manuscrits des pièces, destinés à la censure, ne donnent aucune précision sur ces éléments qui ne l'intéressent pas, et les programmes ou les comptes rendus des journaux ne s'attardent pas à décrire ce qui défie d'ailleurs la description. On peut cependant émettre I'hypothèse qu'elles constituent les ressorts comiques majeurs de ces pièces dont l'argument est tout de même assez mince et dont les dialogues sont assez peu développés.

Bien avant de devenir le principe de très nombreux films comiques, la poursuite et les cascades qui lui sont associées constituent donc un procédé comique dominant des pantomimes de cirques. Mais plus qu'un procédé, et plus que des figures particulières, c'est la forme même du comique des pantomimes, effréné, physique, absurde que le premier cinéma semble avoir adopté. Les bandes tournées par Foottit et Chocolat, à cause de leur brièveté toute cinématographique, donnent sans doute une idée trompeuse de leurs prestations ; elles permettent

$43 \mathrm{Ibid}$.

44 Gribouille, pantomime, Nouveau Cirque, 1891, Archives nationales F 181327.

45 Le Roi Dagobert, pantomime, Nouveau Cirque, 1891, Archives nationales F 181327.

46 América, op. cit.

47 Pierrot soldat, pantomime, Nouveau Cirque, 1895, Archives nationales F 181327.

48 La Reine de Bercy, fantaisie nautique, Nouveau Cirque, 1895, Archives nationales F 181327.

49 La Petite Plage, fantaisie nautique, Nouveau Cirque, 1895, Archives nationales F 181327.

50 Le Feu au moulin, op. cit.

51 Paris Pékin, bouffonnerie nautique, Nouveau Cirque, 1896, Archives nationales F 181327.

52 Pierrot aux Enfers, op. cit.

53 Les Cent Kilos, bouffonnerie nautique, Nouveau Cirque, 1897, Archives nationales F 181327.

54 Paris-Poursuites, Nouveau Cirque, 1903, Archives nationales F 18 1327. C'est aux poursuites judiciaires, notamment à celles dont fait alors l'objet la célèbre Thérèse Humbert, que le titre renvoie ; on peut néanmoins se demander s'il n'a pas été choisi pour son caractère équivoque.

55 Paris qui trotte, op. cit. 
$1895 /$

au moins d'entrevoir ce comique rythmé, haletant, imprévu, volontiers violent, méchant, cynique avec une espèce d'innocence, qui crée cette " atmosphère de nursery du diable »56 dont parle Cocteau à propos de Foottit. Or, dans les pantomimes du Nouveau Cirque, toute cette frénésie conduit ordinairement à la catastrophe, mais à une catastrophe enjouée qui, loin de mener à la mort, régresse vers l'enfance. À la fin d'América, un train, « un véritable train avec sa locomotive marchant la vapeur, ses wagons, son fourgon des bagages "57 s'engage sur un pont qui " s'effondre entraînant dans l'eau les wagons, les voyageurs et les bagages. - Heureusement, des secours sont promptement organisés et tout le monde est sauvé " ${ }^{58}$. La catastrophe ferroviaire, loin de se solder par des pertes humaines ${ }^{59}$, tourne au joyeux bain collectif.

Le Nouveau Cirque présente en effet cette particularité d'avoir été conçu pour être transformable en piscine. Ce dispositif est utilisé pour les pantomimes, qui sont donc généralement des « pantomimes nautiques " 60 . À I'issue de la première partie, sous les yeux des spectateurs, en quelques minutes, le tapis en fibres de coco est roulé, le plancher s'abaisse, l'eau jaillit et remplit le bassin qui s'est formé, puis des décors se dressent et la pantomime commence. De ces instants, Cocteau gardait un souvenir ébloui, qui, étrangement, faisait pâlir à ses yeux le cinéma lui-même : "Le clou du programme était la pantomime nautique. L'arrivée de l'eau me laisse un regret poignant. Aucun truc, aucun gag de cinématographe ne remplacera cette merveille ${ }^{61}$.

Et c'est dans l'eau, dans l'heureuse confusion de la baignade et du barbotage, qui conclut souvent une ultime poursuite, que bien des pantomimes du Nouveau Cirque s'achèvent, en un final largement improvisé. Ainsi, la Grenouillère (1886) : " À la fin, Cascades et Baignade générale ad libitum »62; Gribouille (1891) : «Inondation de la piste, barbotage général. Cascades. Final „63 ; le Roi Dagobert (1891) : "Mais aussitôt la piste s'enfonce, la table rentre sous l'eau et ils [le roi, les valets] restent à barboter pendant la sortie finale "64; la Rosière de

56 Jean Cocteau, Portraits-souvenir, 1935, repris dans Romans/poésies, poésie critique, théâtre/cinéma, Paris, Librairie générale française, coll. La Pochothèque, 1995, p. 763.

57 América, op. cit.

58 lbid.

59 Cette catastrophe ferroviaire pour rire est sans doute un souvenir de celle, tristement célèbre, d'Ashtabula (Ohio), qui, consécutive à l'effondrement d'un pont, avait fait, en 1876, plusieurs dizaines de morts.

60 Le succès du dispositif du Nouveau Cirque incita des cirques itinérants, comme le cirque Marcoud ou le cirque Plège, à l'imiter (voir par exemple à ce sujet les documents reproduits en fac-similé dans Robert Barrier, Grand répertoire illustré des cirques en France, 1845-1995, Crépy-en-Valois, Robert Barrier, 1995, pp. 160 et 200).

61 Jean Cocteau, op. cit., p. 763.

62 Raoul Donval, la Grenouillère, 1886, op. cit., p. 15.

63 Gribouille, op. cit.

64 Le Roi Dagobert, op. cit. 
Charenton (1893) : " Les gendarmes, l'adjoint, les pompiers, se mettent à [la] poursuite [de Foottit et Chocolat] par le même chemin [une corde tendue au-dessus de l'eau] pendant que la tribune officielle sciée traîtreusement par l'infâme Chocolat, s'effondre dans l'eau »65 ; le Moulin du Gué (1894) : « Le sergent en tête, [les soldats] poursuivent les réfractaires. Tous glissent par la planche du moulin. Cascades et Baignade générale "66; la Reine de Bercy (1895) : « Puis on prépare le mât de cocagne. Les concurrents s'approchent en bateau et commencent à grimper. Mais tout à coup le mât s'incline. Le feu d'artifice part subitement. Cris. Clameurs. Cascades et Baignade générale " ${ }^{67}$; la Petite Plage (1895) : « Le bain. Les jeunes filles se jettent à l'eau. Au milieu du bain apparaît un phoque. Un gendarme le poursuit. Cascades. Tout le monde a peur et se sauve. Poursuite. Cascades et baignade générales "68; l'île des bossus (1896) : "Combat naval dirigé par Coussinet dans sa barque. Cascades et baignades générales "69; le Feu au moulin (1896) : "Cascades et baignade générales "70; les Cent Kilos (1897) : «Bousculades. Cris. Un énorme 100 kilos flotte à la surface. D’autres veulent monter sur le bateau qui coule. Cascades. Baignade générale »71.

Cette figure de la catastrophe régressive n'est-elle pas aussi au cœur du premier cinéma comique, de tant de films de poursuite, de tant d'aventures de Calino, de Zigoto ou d'Onésime, quand l'ordre ordinaire bascule incessamment dans le chaos ? Certes, au cinéma, la chute dans le désordre est un procédé itératif, quand elle est plutôt terminale au cirque, et si l'eau en est parfois le réceptacle, c'est loin d'être systématique. Tout de même, il semble bien qu'on doive établir un parallèle entre ces plongeons dans l'indifférencié qui enchantaient le public des années 1890 et ces effondrements innocents, ces infantiles anéantissements cinématographiques. Et sans doute y a-t-il bien des écarts, qu'il n'était pas dans mon propos de relever ici, entre le premier cinéma comique français et la pantomime de cirque. On conviendra pourtant qu'à certains égards ces deux spectacles sont si proches que l'un pourrait sembler le prolongement de l'autre.

65 La Rosière de Charenton, pantomime nautique, Nouveau Cirque, 1893, Archives nationales F 181327. 66 Le Moulin du gué, fantaisie nautique, Nouveau Cirque, 1894, Archives nationales F 181327. 67 La Reine de Bercy, op. cit.

68 La Petite Plage, op. cit.

69 L'île des bossus, pantomime nautique, Nouveau Cirque, 1896, Archives nationales F 181327.

70 Le Feu au moulin, op. cit.

71 Les Cent kilos, op. cit. 